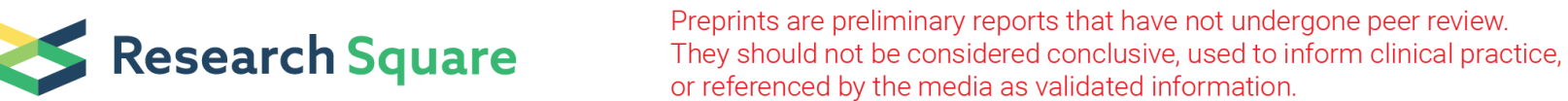

\section{Mesorectal Excision With Or Without Lateral Pelvic Lymph Node Dissection For Mid-Low Rectal Cancer: Efficacy and Prognostic Analysis}

\section{Sicheng Zhou}

National Cancer Center, Chinese Academy of Medical Sciences and Peking Union Medical College Yujuan Jiang

National Cancer Center, Chinese Academy of Medical Sciences and Peking Union Medical College Wei Pei

National Cancer Center, Chinese Academy of Medical Sciences and Peking Union Medical College Jianwei Liang ( $\square$ Liangjw1976@163.com )

National Cancer Center, Chinese Academy of Medical Sciences and Peking Union Medical College Zhixiang Zhou

National Cancer Center, Chinese Academy of Medical Sciences and Peking Union Medical College

\section{Research Article}

Keywords: Mesorectal Excision, Lateral pelvic lymph node dissection, Prognosis

Posted Date: June 10th, 2021

DOl: https://doi.org/10.21203/rs.3.rs-579455/v1

License: (c) (i) This work is licensed under a Creative Commons Attribution 4.0 International License. Read Full License 


\section{Abstract \\ Background}

The efficacy of lateral pelvic lymph node dissection (LPND) for mid-low rectal cancer and the prognosis of patients with lateral pelvic lymph node metastasis (LPNM) remain controversial. The goal of this study was to evaluate the efficacy of LPND for stage II or III middle-low rectal cancer and investigate the impact of LPNM on survival outcomes.

\section{Methods}

Data from 858 consecutive patients who were diagnosed with stage II-III mid-to-low rectal cancer and underwent curative resection were reviewed. After propensity matching, 125 matched pairs were selected and divided into the total mesorectal excision (TME) group and TME + LPND group for evaluation. According to the location of lymph node (LN) metastasis, 250 patients were divided into the N0 group, mesorectal-LN group and LPN group. In addition, the survival data of 100 patients with stage IV rectal cancer who underwent curative resection were also reviewed to compare prognostic differences.

\section{Results}

The prognosis of patients in the TME + LPND group did not improve significantly in terms of local recurrence rate ( $4.8 \%$ vs. $4.0 \%, P=0.758)$, distant metastasis rate $(18.4 \%$ vs. $18.4 \%, P=1.000)$ or 5 -year recurrence-free survival (RFS) $(63.1 \%$ vs. $66.2 \%, P=0.269)$. Overall, $89(35.6 \%), 129(51.6 \%)$, and 22 $(8.8 \%)$ patients were classified into the NO, mesorectal-LN, and LPN groups, respectively. When the mesorectal LN and LPN groups were subdivided, RFS was not significantly different between the internal LPN and N1 groups $(70.8 \%$ vs. $62.5 \%, P=0.905)$. There was no significant difference in RFS between the external group and the stage IV group ( $31.6 \%$ vs. $22.5 \%, P=0.302)$, but RFS in the former group was significantly worse than that in the N2 group ( $31.6 \%$ vs. $40.4 \%, P=0.044)$.

\section{Conclusion}

No differences were observed regarding any recurrence or RFS between the TME group and TME + LPND group. Although LPNM was an independent poor prognostic factor for RFS, patients limited to internal iliac LN metastasis appeared to achieve a survival benefit from LPND and could be regarded as regional LN metastasis. Patients with LPNM other than the internal iliac $L N$ had a poor prognosis; the prognosis was significantly worse than that of N2 and slightly better than that of stage IV.

\section{Introduction}


Mesorectal excision (ME) or total mesorectal excision (TME), that is, removal of the mesorectal tissue at least $4 \mathrm{~cm}$ from the distal end of the tumour, is currently an internationally recognized surgical principle for low rectal cancer. However, approximately $7 \%-23 \%$ of patients with stage II or III middle-low rectal cancer occasionally develop metastases to the lateral pelvic lymph nodes (LPNs), which are outside the scope of surgical dissection of the TME or ME and are associated with poor prognosis and higher local recurrence rates ${ }^{[1-5]}$. In Japan, LPNs are regarded as regional lymph nodes, which are considered within the scope of the N3 stage. The JCOG (Japanese Clinical Oncology Group) 0212 trial demonstrated the safety and oncology efficacy of LPN dissection (LPND) ${ }^{[1,2]}$. The results of this trial revealed that TME + LPND has satisfactory perioperative outcomes, with similar postoperative morbidity and mortality as TME alone. Furthermore, TME with LPND resulted in a lower local recurrence, especially in the lateral pelvis, compared to ME alone. Therefore, the JSCCR guidelines suggested that TME + LPND should be performed routinely for patients with stage II/III middle-low rectal cancer ${ }^{[6]}$.

In contrast, several studies, also from Japan, suggested that the overall benefit related to local control and survival of LPND is not promising in patients with LPN metastasis (LPNM) ${ }^{[4,7-10]}$. We suggest that there are two reasons for the difference in the results reported above. First, only surgeons who specialized in TME + LPND participated in the JCOG trial. Therefore, this technically demanding procedure may yield different survival outcomes in different institutions. More importantly, the JCOG trial excluded almost $20 \%$ of patients with clinically suspected LPNM diagnosed by preoperative imaging from the study, which is not in line with the real situation in clinical practice. Therefore, it is necessary to clarify the effectiveness of TME + LPND with regard to increasing local control and prolonging survival for patients with LPNM in different regions and institutions. In addition, we found that patients with LPNM located in the area of the internal iliac tended to achieve a better prognosis. The present study aimed to determine the safety and oncological efficacy of TME + LPND for middle-low rectal cancer patients in the National Cancer Center and to analyse the prognosis of LPNM localized to or extending beyond the internal iliac area.

\section{Patients And Methods}

\section{Patients}

We reviewed the records of 2372 patients with rectal cancer who underwent curative resection at the National Cancer Center/National Clinical Research Center for Cancer/Cancer Hospital, Chinese Academy of Medical Sciences and Peking Union Medical College from January 2015 and January 2020. The inclusion criteria were as follows: (1) 18-75 years old; (2) lower margin of rectal tumour below the peritoneal reflection; (2) pathological diagnosis of stage II-III; and (3) pathological diagnosis of adenocarcinoma. Patients who underwent transanal local excision or had a history of other malignant tumours were excluded from the study. Thus, 883 patients with stage II-III mid-low rectal cancer were included and divided into the TME + LPND group and the TME group according to the surgical methods. All enrolled patients were included in the propensity score matching (PSM) process, and 125 matched 
pairs were eventually selected (Fig. 1). The study protocol was approved by the Ethics Committee of the Cancer Hospital, Chinese Academy of Medical Sciences (NCC 2017-YZ-026, Oct 17, 2017), and written informed consent was obtained from all enrolled patients.

Preoperative investigations for all patients included laboratory examination, endoscopy, pelvic magnetic resonance imaging (MRI), and computed tomography (CT) of the abdomen. All procedures were performed by surgeons with more than 20 years of experience in colorectal surgery. At our institution, there is no standard indication for LPND. The surgical method was ascertained at the discretion of the surgeon with full consideration of the patient's characteristics, pathological results and preoperative examination. The American Joint Committee on Cancer (AJCC) staging Manual (8th edition) was used for tumour staging ${ }^{[11]}$. According to the JSCCR classification, LPN areas were divided into six regions: obturator, external iliac, internal iliac, common iliac, aortic bifurcation, and median sacral regions ${ }^{[12]}$. Patients were divided into three groups according to the status of lymph node $(L N)$ metastasis: (1) patients without any LN metastasis were classified into the NO group; (2) patients with mesenteric LN metastasis without LPNM were classified into the mesorectal LN group; and (3) patients with LPNM, regardless of mesenteric LN status, were divided into the LPN group.

Patients were followed-up through outpatient clinic or telephone after operation, including survival, causes of death and the follow-up period. The follow-up deadline was the recurrence date or February 1 , 2021, whichever came first. Serum tumour markers, abdominal CT examinations, and colonoscopy were performed every 3 years for the first 3 years and every 6 months for the next 2 years. The long-term endpoint of this study was 5-year recurrence-free survival (RFS), and the data were collected based on this follow-up survey.

\section{Statistical analysis}

Patients in the TME group were matched in a 1:1 ratio to those in the TME + LPND group through PSM based on the following factors: age, sex, BMI, ASA score, preoperative CEA level, preoperative CA19-9 level, distance from AV, histological type, pathological T stage, mesorectal LN, status of LN metastasis, LPLNs harvested, mesorectal LNs harvested, lymphatic invasion, perineural invasion and adjuvant therapy.

Clinical and pathological data are expressed as frequencies and percentages or means \pm standard deviations. The differences in variables between the two groups were measured by the $\chi^{2}$ test or Fisher's exact test. The RFS curves were calculated by the Kaplan-Meier method and compared by the log-rank test. The predictors determined to have a $P$ value $<0.20$ in univariate analysis were subsequently tested by multivariate analysis through a Cox proportional hazards model, and hazard ratios (HRs) with a 95\% confidence interval $(\mathrm{Cl})$ were reported for each variable. A P value less than 0.05 was considered statistically significant. Data statistics and analysis were performed using SPSS for Windows version 20.0 (SPSS, Chicago, Illinois, USA).

\section{Results}




\section{Patient characteristics}

A total of 125 marched pairs were selected through propensity scoring and enrolled in the study. The baseline data, clinical parameters and pathological characteristics are presented in Table 1. After matching, the TME group and TME + LPND group were well balanced in terms of age, sex, BMI, ASA score, preoperative CEA level, preoperative CA19-9 level, distance from AV, histological type, pathological T stage, mesorectal LN, status of LN metastasis, LPLNs harvested, mesorectal LNs harvested, lymphatic invasion, perineural invasion and adjuvant therapy. 
Table 1

Clinical and pathological characteristics of rectal cancer patients who underwent TME with or without LPND

\begin{tabular}{|c|c|c|c|}
\hline Variables & $\begin{array}{l}\text { TME } \\
(n=125)\end{array}$ & $\begin{array}{l}\text { TME + LPND } \\
(n=125)\end{array}$ & $P$ \\
\hline Gender & & & 0.798 \\
\hline Male & $71(56.8)$ & $73(58.4)$ & \\
\hline Female & $54(43.2)$ & $52(41.6)$ & \\
\hline Age (years) & & & 0.899 \\
\hline$<60$ & $68(54.4)$ & $69(55.2)$ & \\
\hline$\geq 60$ & $57(45.6)$ & $56(44.8)$ & \\
\hline $\mathrm{BMI}\left(\mathrm{Kg} / \mathrm{m}^{2}\right)$ & $24.5 \pm 3.1$ & $25.0 \pm 3.1$ & 0.226 \\
\hline ASA score & & & 0.625 \\
\hline$|-| I$ & $115(92.0)$ & $117(91.4)$ & \\
\hline III & $10(8.0)$ & $8(8.6)$ & \\
\hline Preoperative CEA level (ng/ml) & $9.4 \pm 22.2$ & $10.3 \pm 20.5$ & 0.755 \\
\hline Preoperative CA19-9 level (ng/ml) & $24.5 \pm 41.1$ & $21.2 \pm 34.3$ & 0.511 \\
\hline Distance from AV (cm) & $5.4 \pm 1.5$ & $5.1 \pm 1.4$ & 0.452 \\
\hline Histological type & & & 0.172 \\
\hline Well/moderate & $81(64.8)$ & $91(72.8)$ & \\
\hline Other & $44(35.2)$ & $34(27.2)$ & \\
\hline \multicolumn{4}{|l|}{ Pathological T stage } \\
\hline T1-T2 & $34(27.2)$ & $31(24.8)$ & \\
\hline T3-T4 & $91(72.8)$ & $94(75.2)$ & \\
\hline Mesorectal LN & & & 0.964 \\
\hline No & $50(40.0)$ & $51(40.8)$ & \\
\hline N1 & $43(34.4)$ & $41(32.8)$ & \\
\hline N2 & $32(25.6)$ & $33(26.4)$ & \\
\hline Status of LN metastasis & & & - \\
\hline NO & $50(40.0)$ & $49(39.2)$ & \\
\hline
\end{tabular}




\begin{tabular}{|llll|}
\hline Variables & $\begin{array}{l}\text { TME } \\
(\mathbf{n}=\mathbf{1 2 5})\end{array}$ & $\begin{array}{l}\text { TME + LPND } \\
(\mathbf{n}=\mathbf{1 2 5})\end{array}$ & $\boldsymbol{P}$ \\
\hline Mesorectal-LN & $75(60.0)$ & $54(43.2)$ & \\
\hline LPN & $0(0)$ & $22(17.6)$ & \\
\hline LPLNs harvested & - & $9.6 \pm 5.7$ & - \\
\hline Mesorectal LNs harvested & $16.9 \pm 10.4$ & $17.7 \pm 10.2$ & 0.755 \\
\hline Lymphatic invasion & $35(28.0)$ & $34(27.2)$ & 0.887 \\
\hline Perineural invasion & $48(38.4)$ & $45(36.0)$ & 0.665 \\
\hline Adjuvant therapy & $83(66.4)$ & $80(64.0)$ & 0.690 \\
\hline
\end{tabular}

\section{Operative Detail And Postoperative Complications}

The perioperative outcomes of the patients are summarized in Table 2. Low anterior resection and abdominoperineal resection were performed in 107 (21.4\%) and 137 patients (27.4\%), respectively. Thirty (24.0\%) patients in the TME + LPND group underwent bilateral LPND. TME with LPND required a significantly longer operation time (356.1 vs. 244.8 minutes, $P<0.001)$ and resulted in a similar estimated blood loss (78.7 vs. $64.1 \mathrm{ml}, P=0.202)$ than TME alone. In terms of postoperative complications, there was no significant difference between the two groups (16.0 vs. 12.0, $P=0.362)$, and each complication was similar. The time to first flatus ( 3.1 vs. 3.3 days, $P=0.552)$ and the postoperative hospital stay ( 8.5 vs. 8.8 days, $P=0.630$ ) were not significantly different between the groups. All patients recovered from surgery and were discharged from the hospital, and no deaths were observed during the perioperative period in either group. 
Table 2

Perioperative detail of rectal cancer patients who underwent TME with or without LPND

\begin{tabular}{|llll|}
\hline Variables & TME & TME + LPND & P \\
& $(\mathbf{n = 1 2 5 )}$ & $(\mathbf{n = 1 2 5 )}$ & \\
\hline Types of operation & & & 0.421 \\
\hline Low anterior resection & $57(45.6)$ & $50(40.0)$ & \\
\hline Abdominoperineal resection & $64(51.2)$ & $73(58.4)$ & \\
\hline Hartmann procedure & $4(3.2)$ & $2(1.6)$ & \\
\hline LPND & & & - \\
\hline Unilateral dissection & - & $95(76.0)$ & \\
\hline Bilateral dissection & - & $30(24.0)$ & \\
\hline Operative time (min) & $244.8 \pm 60.3$ & $356.1 \pm 76.5$ & $<0.001$ \\
\hline Estimated blood loss (ml) & $64.1 \pm 97.2$ & $78.7 \pm 81.7$ & 0.202 \\
\hline Postoperative complications & $15(12.0)$ & $20(16.0)$ & 0.362 \\
\hline Postoperative bleeding & $3(2.4)$ & $2(1.6)$ & \\
\hline Ileus & $1(0.8)$ & $3(2.4)$ & \\
\hline Anastomosis leakage & $4(3.2)$ & $2(1.6)$ & \\
\hline Pelvic cavity abscess & $5(4.0)$ & $3(2.4)$ & \\
\hline Pneumonia & $3(2.4)$ & $6(4.8)$ & \\
\hline Wound infection & $2(1.6)$ & $5(4.0)$ & \\
\hline Urinary retention & $1(0.8)$ & $2(1.6)$ & \\
\hline Rectovaginal fistula & $1(0.8)$ & $0(0)$ & \\
\hline Time to first flatus (days) & $3.3 \pm 1.2$ & $3.1 \pm 1.4$ & \\
\hline Postoperative hospital stay (days) & $8.8 \pm 5.8$ & $8.5 \pm 5.8$ & \\
\hline Re-operation & $3(2.4)$ & $1(0.8)$ & \\
\hline Mortality & $0(0)$ & $0(0)$ & \\
\hline
\end{tabular}

\section{Local Recurrence And Distant Metastasis}


The median follow-up period of the whole group was 28.0 (range, 3-72) months. Of all 250 patients undergoing curative resection for rectal cancer, 56 (22.4\%) had a recurrence of cancer, including 11 (4.4\%) local recurrence cases and 46 (18.4\%) distant metastasis cases. During the 25-month (range, 2-66) follow-up period, the rates of overall recurrence (23.2 vs. $21.6, P=0.762)$, local recurrence $(4.8 \%$ vs. $4.0 \%$, $P=0.758)$, and distant metastasis $(18.4 \%$ vs. $18.4 \%, P=1.000)$ did not differ between the TME + LPND group and the TME group (Table 3$)$. In addition, the 5 -year RFS rate $(63.1 \%$ vs. $66.2 \%, P=0.269)$ was similar in both groups (Fig. 2).

Table 3

Postoperative recurrence of 250 rectal cancer patients who underwent TME with or without LPND

\begin{tabular}{|llll|}
\hline Variables & $\begin{array}{l}\text { TME } \\
(\mathbf{n}=125)\end{array}$ & $\begin{array}{l}\text { TME + LPND } \\
(\mathbf{n}=125)\end{array}$ & $\boldsymbol{P}$ \\
\hline Overall recurrence (\%) & $27(21.6)$ & $29(23.2)$ & 0.762 \\
\hline Local recurrence & $5(4.0)$ & $6(4.8)$ & 0.758 \\
\hline Distant metastasis & $23(18.4)$ & $23(18.4)$ & 1.000 \\
\hline Liver metastasis & $8(6.4)$ & $14(11.2)$ & \\
\hline Lung metastasis & $18(14.4)$ & $11(8.8)$ & \\
\hline Bone metastasis & $5(4.0)$ & $3(2.4)$ & \\
\hline Peritoneal metastasis & $0(0)$ & $1(0.8)$ & \\
\hline Brain metastasis & $1(0.8)$ & $0(0)$ & \\
\hline Others & $2(1.6)$ & $2(1.6)$ & \\
\hline
\end{tabular}

\section{Prognostic Factors}

To determine prognostic factors for RFS of patients with middle-low rectal cancer, univariate and multivariate regression analyses were performed (Table 4). In univariate analysis, preoperative CEA level $>5 \mathrm{ng} / \mathrm{ml}$, perineural invasion and status of LN metastasis significantly affected RFS $(P<0.05)$. The prognostic factors determined to have a $P$ value $<0.20$ in univariate analysis were subsequently tested by multivariate analysis through a Cox proportional hazards model. As shown in Fig. 3 , the results of multivariate analysis demonstrated that independent prognostic factors associated with RFS were the status of LN metastasis. 
Table 4

Univariate and multivariate analyses for recurrence-free survival of the 250 rectal patients who underwent TME with or without LPND

\begin{tabular}{|c|c|c|c|c|}
\hline \multirow[t]{3}{*}{ Variables } & \multicolumn{4}{|c|}{ Recurrence-free survival } \\
\hline & \multicolumn{2}{|c|}{ Univariate analysis } & \multicolumn{2}{|c|}{ Multivariate analysis } \\
\hline & $\mathrm{HR}(95 \% \mathrm{Cl})$ & $\mathbf{P}$ & $\mathrm{HR}(95 \% \mathrm{Cl})$ & $\mathbf{P}$ \\
\hline Gender: male & $1.36(0.81-2.27)$ & 0.244 & & \\
\hline Age: $\geq 60$ years & $1.08(0.66-1.78)$ & 0.751 & & \\
\hline Preoperative CEA level: > 5ng/ml & $1.70(1.02-2.83)$ & 0.043 & $1.55(0.92-2.61)$ & 0.097 \\
\hline Histology:other & $1.44(0.87-2.39)$ & 0.155 & $1.11(0.62-1.98)$ & 0.721 \\
\hline Lymphatic invasion:yes & $1.55(0.92-2.62)$ & 0.103 & $1.06(0.57-1.97)$ & 0.846 \\
\hline Perineural invasion:yes & $1.75(1.06-2.86)$ & 0.027 & $1.53(0.85-2.76)$ & 0.157 \\
\hline T stage: T3-4 & $1.63(0.87-3.06)$ & 0.130 & $1.09(0.54-2.19)$ & 0.804 \\
\hline \multicolumn{5}{|l|}{ Status of LN metastasis } \\
\hline No & Reference & & Reference & \\
\hline Mesorectal-LN & $1.82(1.01-3.26)$ & 0.045 & $1.95(1.10-3.60)$ & 0.044 \\
\hline LPN & $4.06(1.79-9.20)$ & 0.001 & $3.03(1.23-7.47)$ & 0.016 \\
\hline LPND: yes & $1.32(0.80-2.17)$ & 0.273 & & \\
\hline Adjuvant chemotherapy: yes & $0.94(0.56-1.58)$ & 0.812 & & \\
\hline Postoperative complications: yes & $1.09(0.56-2.15)$ & 0.799 & & \\
\hline
\end{tabular}

Overall, 89 (35.6\%), 129 (51.6\%), and $22(8.8 \%)$ patients were classified into the N0, mesorectal-LN, and LPN groups, respectively. To investigate the prognostic difference between LPNM, regional mesorectal LN metastasis and distant metastasis, the survival of 100 patients with stage IV rectal cancer who underwent curative resection from June 2017 to June 2019 was retrospectively collected and analysed. The 5-year RFS of patients with LPNM was significantly better than that of stage IV patients $(42.0 \% \mathrm{vs}$. $22.5 \%, P=0.033)$ and significantly worse than that of patients with mesorectal LN metastasis ( $42.0 \% \mathrm{vs}$. $59.4 \%, P=0.027$ ) (Fig. 4).

Finally, we subdivided the mesorectal LN group into two groups according to the AJCC tumour staging system (N1: 1-3 regional LN metastasis; N2: >3 regional LN metastasis). The LPN group was also subdivided into two categories (internal-LPN: internal iliac LN metastasis without LN metastasis in the other five lateral pelvic regions; external-LPN: LPN metastasis in the other five lateral pelvic regions, with or without internal iliac LN metastasis). 
The 5-year RFS rates of the N0, N1, internal LPN, N2, external LPN, and stage IV groups were 77.3\%, $70.8 \%, 62.5 \%, 40.4 \%, 31.6$, and $22.5 \%$, respectively (Fig. 5). RFS was not significantly different between the internal-LPN and N1 groups $(P=0.905)$ or between the external-LPN and stage IV groups $(P=0.302)$. Notably, the RFS of the external-LPN group was significantly worse than that of the N2 group $(P=0.044)$.

\section{Discussion}

In the present study, among the 125 patients who underwent TME + LPND, the pathologically confirmed rate of LPNM was $17.6 \%$ (22/125), which is consistent with reporting rates varying from $8.6-18.6 \%$ in the previous literature ${ }^{[8,13,14]}$. In addition, all patients included in this study were operated on by surgeons at the National Cancer Center with more than 20 years of experience in colorectal surgery. Therefore, this study is more in line with the real situation in clinical practice and better provides practice-based evidence.

To minimize the impact of selection bias on the results, PSM was carried out to balance baseline data and clinicopathological characteristics between the two groups. In terms of perioperative outcomes, additional LPND increased the operative time by approximately 113 minutes, which was consistent with a corresponding increase of 106 minutes in the JCOG0212 cohort ${ }^{[1]}$. Regarding surgical safety, there was no marked difference in overall or various postoperative complications between the two groups (16.0\% vs. $12.05, P=0.362)$. In addition, no mortality was noted within 30 days of the perioperative period in either group. Therefore, we suggest that additional LPND on the basis of TME is safe for surgeons with extensive experience in colorectal surgery.

The long-term survival outcomes determine the value and effectiveness of LPND for rectal cancer. However, it has been previously reported that LPND does not significantly improve local control and survival[4,7-10]. A phase III randomized controlled study of 445 patients with stage II/III rectal cancer conducted by Oki and his colleagues showed that LPND had no impact on RFS (HR $=0.941,95 \%$ Cl: $0.696-1.271, P=0.69)$ or overall survival ( $\mathrm{HR}=0.858,95 \% \mathrm{Cl}: 0.601-1.224, P=0.39)$ in all patients $^{[10]}$, which agreed with our results. Moreover, a large multi-centre study conducted by Kobayashi et al. analysed 1272 low rectal cancer patients and showed a similar survival and local recurrence rate for patients with or without LPND. However, considering that LPND was more likely to be performed in patients with advanced tumours, they concluded that LPND may be beneficial for specific patients ${ }^{[4]}$. Our study balanced baseline data, such as pathological features, $\mathrm{T}$ and $\mathrm{N}$ stage, between the two groups to reduce selection bias. The results demonstrated that there was no significant reduction in the local recurrence rate $(4.8 \%$ vs. $4.0 \%, P=0.758)$ or distant metastasis rate $(18.4 \%$ vs. $18.4 \%, P=1.000)$ in the TME + LPND group. In addition, the 5 -year RFS rate $(63.1 \%$ vs. $66.2 \%, P=0.269)$ was also similar in both groups. Therefore, we suggest that LPND does not confer a survival benefit for all patients with lowmiddle rectal cancer.

It is well known that LPNM is associated with local recurrence and poor long-term survival. A retrospective study involving 149 patients conducted by Sato et al. proved that patients with LPNM were 
more likely to relapse, and LPNM was an adverse prognostic factor for patients with rectal carcinoma below the peritoneal reflection ${ }^{[7]}$. In the present study, we explored prognostic factors in 250 middle-low rectal cancer patients who underwent curative resection, and the results revealed that mesorectal LN metastasis (HR: 1.95; 95\% Cl, 1.10-3.60; $P=0.044$ ) and LPNM (HR: $3.03 ; 95 \% \mathrm{Cl}, 1.23-7.47 ; P=0.016$ ) were independent poor predictive factors affecting RFS, which agrees with the data previously reported in the literature ${ }^{[7,10]}$.

Moreover, we investigated and compared the prognostic differences between LPNM, regional mesorectal LN metastasis and distant metastasis. The results demonstrated that the RFS rate of patients with LPNM was significantly better than that of stage IV patients $(P=0.033)$ and significantly worse than that of patients with mesorectal $L N$ metastasis $(P=0.027)$. Previous literature has reported that LPND may provide survival benefits for patients with LPNM in the internal iliac vessel region or the obturator region ${ }^{[5}$, $7,15,16]$. A Japanese nationwide multi-institutional study enrolled 11,567 patients with stage I-III rectal cancer and revealed that both the overall survival (OS) and cancer-specific survival (CCS) of patients with internal LPN ( $P=0.9585$ for OS and 0.5742 for CSS) and external LPN metastases ( $P=0.3342$ for OS and 0.4347 for CSS) were similar to those of patients with N2a and N2b stages, respectively. Furthermore, both the OS and CSS of the patients with external LPN metastasis were significantly better than those with stage IV metastasis. ( $P=0.024$ for OS and 0.011 for CSS $)^{[5]}$. In the present study, we further subdivided each group and found that RFS was not significantly different between the internal-LPN and N1 groups $(P=0.905)$. However, in contrast to the literature reported above, our results showed that the RFS of patients with external LPN metastasis was not significantly different from that of patients with stage IV rectal cancer $(P=0.302)$ and was significantly lower than that of patients with stage N2 rectal cancer $(P=0.044)$. We suggested that the 5 -year rate of RFS in patients with external LPN metastasis is approximately $10 \%$ higher than that in stage IV rectal patients (31.6\% vs. $22.5 \%)$, possibly due to the small sample size, which could not achieve a significant difference. We did not further subdivide patients by $\mathrm{N} 2$ stage, which made it impossible to describe in detail the difference in prognosis between LPNM and mesorectal LN metastasis.

Several limitations of the present study should be clarified and considered. The first potential limitation involves the selection bias caused by the retrospective nature. Theoretically, patients selected for LPND may have advanced disease and a higher rate of LPNM. However, we performed PSM to balance the cohort as far as practicable. Second, the sample size of this study was small, and only 350 patients with rectal cancer were included for discussion and analysis. Therefore, a multi-centre randomized controlled trial is needed to further verify our conclusions.

In conclusion, no differences were observed regarding any recurrence or RFS between the TME group and TME + LPND group. Although LPNM was an independent poor prognostic factor for RFS, patients limited to internal iliac LN metastasis appear to achieve a survival benefit from LPND and can be regarded as regional LN metastasis. Patients with LPNM other than the internal iliac LN have a poor prognosis that is significantly worse than that of N2 and slightly better than that of stage IV.

Page $12 / 18$ 


\section{List Of Abbreviations}

Lateral pelvic lymph node dissection (LPND); Lateral pelvic lymph node metastasis (LPNM); Total mesorectal excision (TME); Lymph node (LN); Recurrence-free survival (RFS); Mesorectal excision (ME); Pelvic magnetic resonance imaging (MRI); Computed tomography (CT); American Joint Committee on Cancer (AJCC); Hazard ratios (HRs); Confidence interval (Cl);

\section{Declarations}

\section{Ethics approval and consent to participate}

The ethics committee of the National Cancer Center/Cancer Hospital, Chinese Academy of Medical Sciences and Peking Union Medical College approved this study (NCC 2017-YZ-026, Oct 17, 2017). Prior written informed consent was obtained from all study participants. All methods were performed in accordance with the Declaration of Helsinki.

\section{Availability of data and materials}

The datasets generated and/or analysed during the current study are not publicly available due to the data is confidential patient data but are available from the corresponding author on reasonable request.

\section{Consent to publish}

Not Applicable.

\section{Competing interests}

The authors declare that they have no competing interests.

\section{Funding}

This work was supported by the Capital's Funds for Health Improvement and Research (2016-2-4022). The funders had no role in the design of the study, data collection, analysis, and manuscript writing.

\section{Authors' contributions}

Contributions: (I) conception and design: JWL and SCZ; (II) administrative support: JWL and ZXZ; (III) provision of study materials or patients: YJJ and SCZ ; (IV) collection and assembly of data: WP and ZXZ; (V) data analysis and interpretation: JWL and SCZ. All authors read and approved the final manuscript.

\section{Acknowledgements}

Not applicable.

\section{References}


1. Fujita S, Akasu T, Mizusawa J, et al. Postoperative morbidity and mortality after mesorectal excision with and without lateral lymph node dissection for clinical stage II or stage III lower rectal cancer (JCOG0212): results from a multicentre, randomised controlled, non-inferiority trial. Lancet Oncol. 2012;13(6):616-21.

2. Fujita S, Mizusawa J, Kanemitsu Y, et al. Mesorectal Excision With or Without Lateral Lymph Node Dissection for Clinical Stage II/III Lower Rectal Cancer (JCOG0212): A Multicenter, Randomized Controlled, Noninferiority Trial. Ann Surg. 2017;266(2):201-207.

3. Hashiguchi Y, Muro K, Saito Y, et al. Japanese Society for Cancer of the Colon and Rectum (JSCCR) guidelines 2019 for the treatment of colorectal cancer. Int J Clin Oncol. 2020;25:1-42.

4. Kobayashi $\mathrm{H}$, Mochizuki $\mathrm{H}$, Kato $\mathrm{T}$, et al. Outcomes of surgery alone for lower rectal cancer with and without pelvic sidewall dissection. Dis Colon Rectum. 2009;52(4):567-76.

5. Akiyoshi T, Watanabe T, Miyata S, et al. Results of a Japanese nationwide multi-institutional study on lateral pelvic lymph node metastasis in low rectal cancer: is it regional or distant disease? Ann Surg. 2012;255(6):1129-34.

6. Yagi R, Shimada $\mathrm{Y}$, Kameyama $\mathrm{H}$, et al. Clinical significance of extramural tumor deposits in the lateral pelvic lymph node area in low rectal cancer: a retrospective study at two institutions. Ann Surg Oncol. 2016;23(Suppl 4):S552『S558.

7. Sato $\mathrm{H}$, Maeda K, Maruta M. Prognostic significance of lateral lymph node dissection in node positive low rectal carcinoma. Int J Colorectal Dis. 2011;26(7):881-9.

8. Wang L, Hirano Y, Heng G, et al. The Significance of Lateral Lymph Node Metastasis in Low Rectal Cancer: a Propensity Score Matching Study. J Gastrointest Surg. 2020; Epub ahead of print.

9. Numata $\mathrm{M}$, Tamagawa $\mathrm{H}$, Kazama $\mathrm{K}$, et al. Lateral lymph node dissection for mid-to-low rectal cancer: is it safe and effective in a practice-based cohort? BMC Surg. 2021;21(1):51.

10. Oki E, Shimokawa M, Ando K, et al. Effect of lateral lymph node dissection for mid and low rectal cancer: an ad-hoc analysis of the ACTS-RC (JFMC35-C1) randomized clinical trial. Surgery. 2019;165:586-92.

11. Amin MB, Edge S, Greene F, et al. AJCC cancer staging manual. 8th ed. New York: Springer; 2017.

12. Japanese Society for Cancer of the Colon and Rectum. Japanese Classification of Colorectal Carcinoma. 1st English ed. Tokyo, Japan: Kanehara \& Co; 1997.

13. Ishihara S, Kawai K, Tanaka T, et al. Oncological Outcomes of Lateral Pelvic Lymph Node Metastasis in Rectal Cancer Treated With Preoperative Chemoradiotherapy. Dis Colon Rectum 2017;60(5):469476.

14. Nagasaki T, Akiyoshi T, Fujimoto Y, et al. Preoperative Chemoradiotherapy Might Improve the Prognosis of Patients with Locally Advanced Low Rectal Cancer and Lateral Pelvic Lymph Node Metastases. World J Surg 2017;41:876-883.

15. Yokoyama S, Takifuji K, Hotta T, et al. Survival benefit of lateral lymph node dissection according to the region of involvement and the number of lateral lymph nodes involved. Surg Today 2014;44(6):1097-103. 
16. Ueno $\mathrm{H}$, Mochizuki $\mathrm{H}$, Hashiguchi $\mathrm{Y}$, et al. Potential prognostic benefit of lateral pelvic node dissection for rectal cancer located below the peritoneal reflection. Ann Surg 2007;245(1):80-7.

\section{Figures}

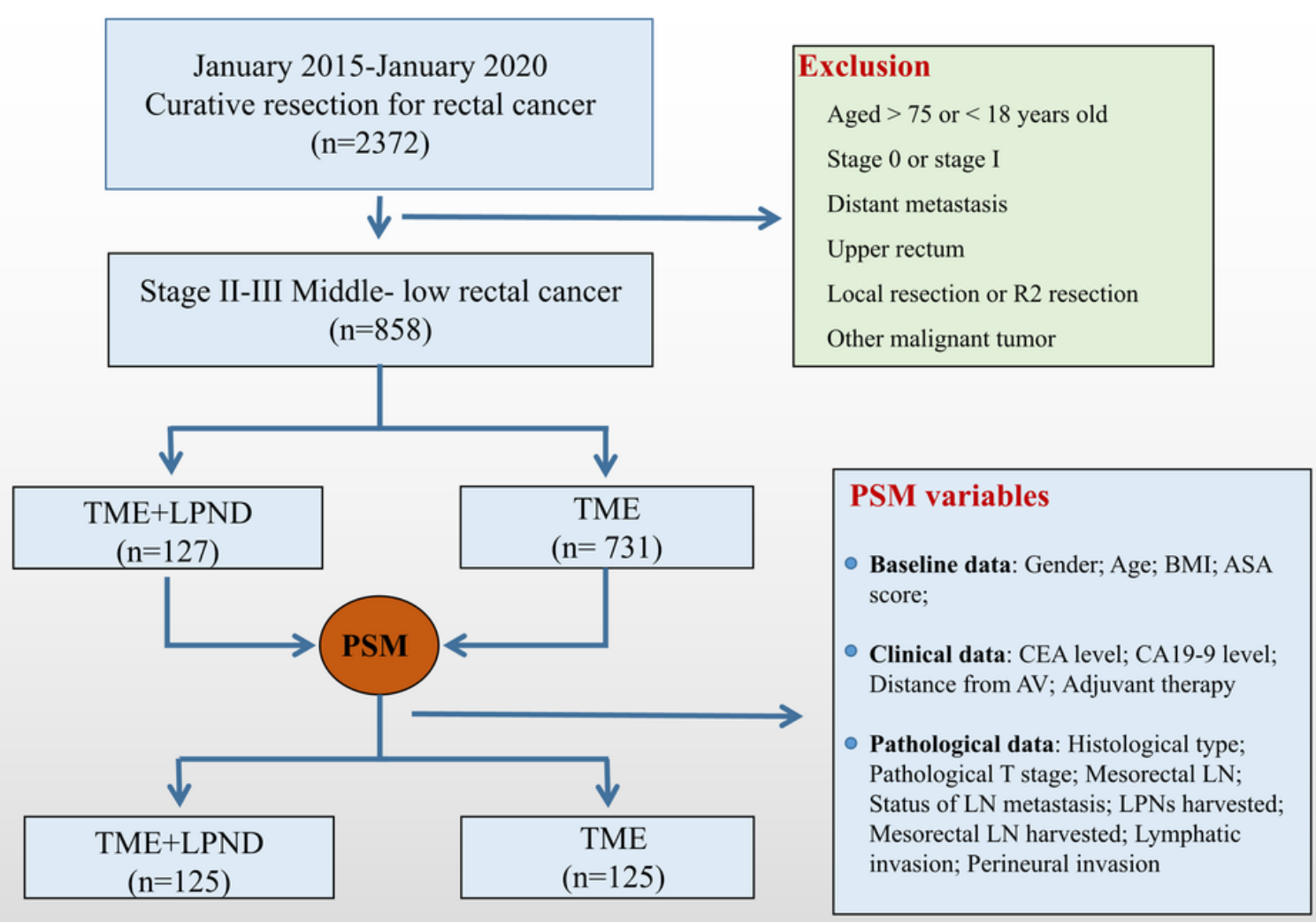

Figure 1

Group flow chart 


\section{Recurrence-free survival}

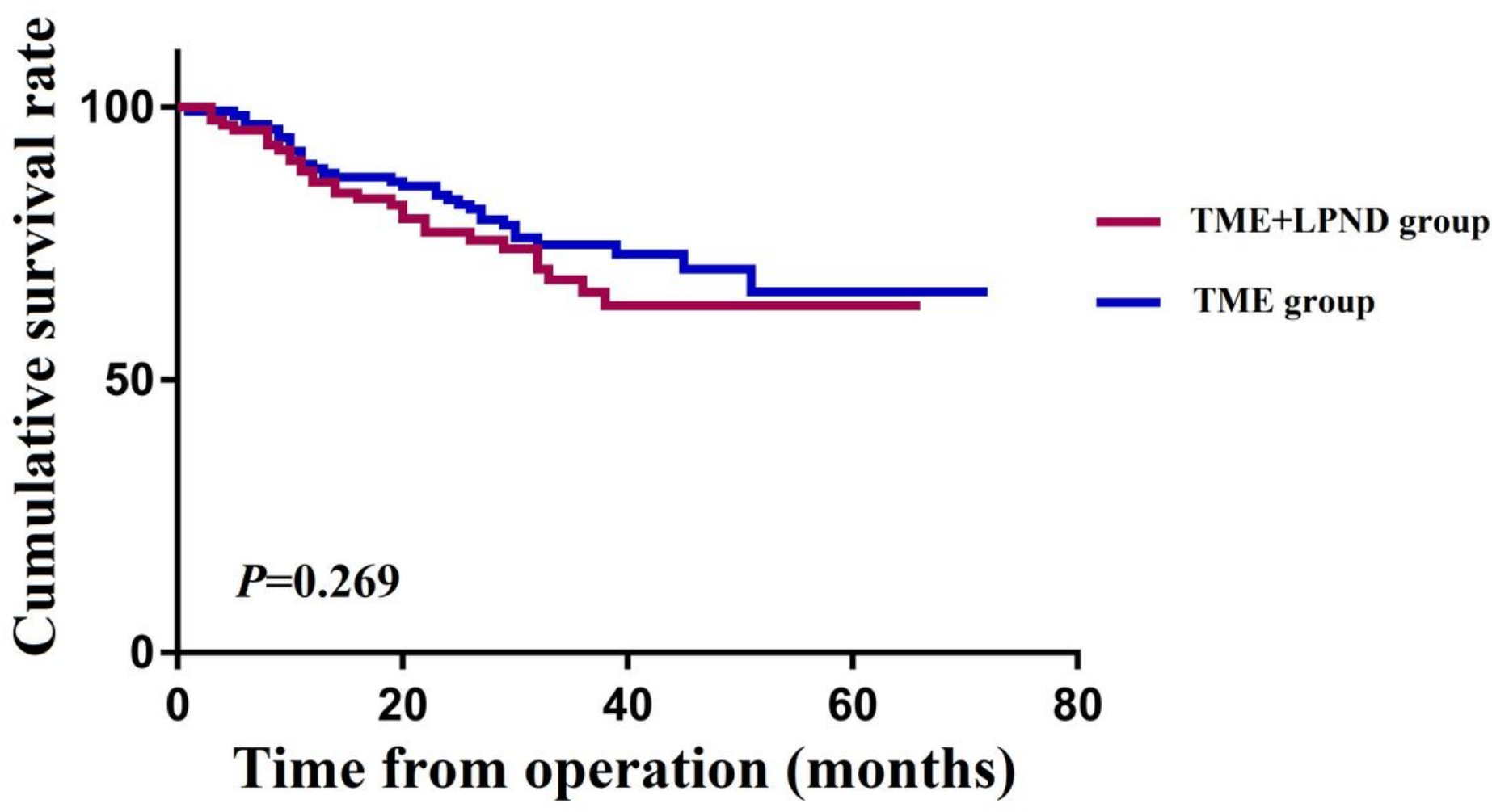

Figure 2

Recurrence-survival rate of rectal patients in the TME+LPND group and TME group

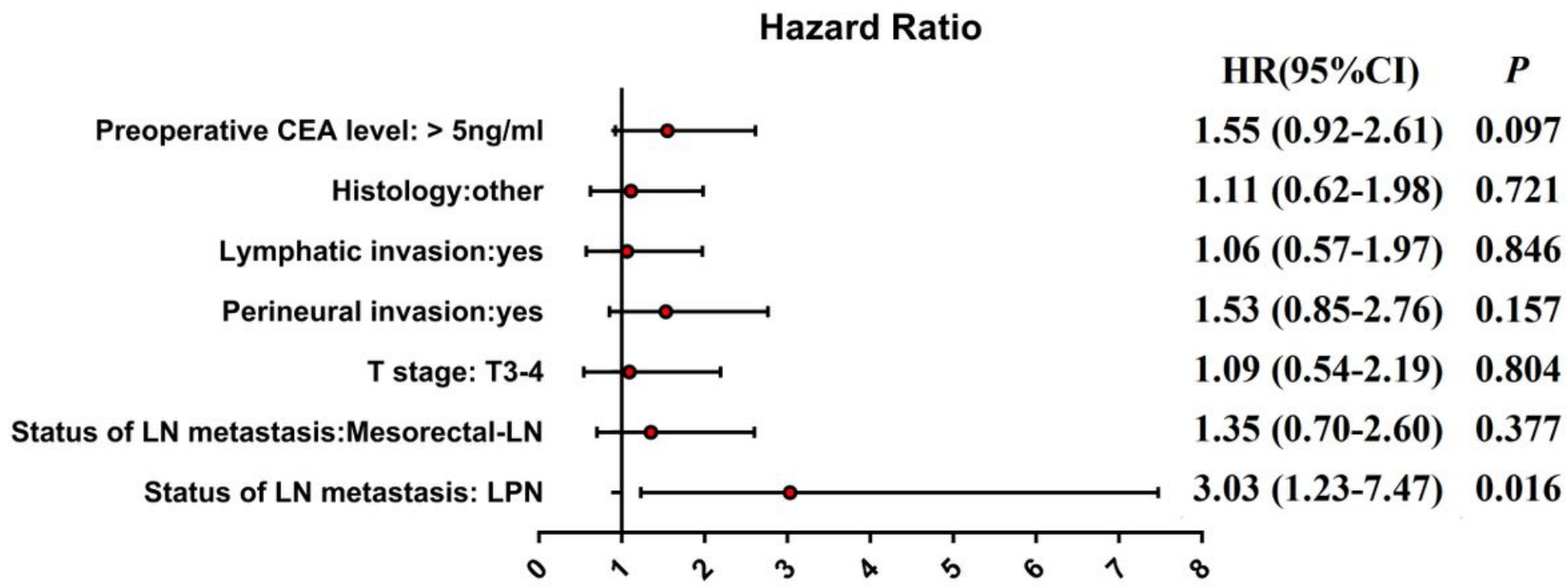

Figure 3

Forest plots for recurrence-free survival of rectal cancer patients who underwent TME with or without LPND based on multivariable COX proportional hazard model. 


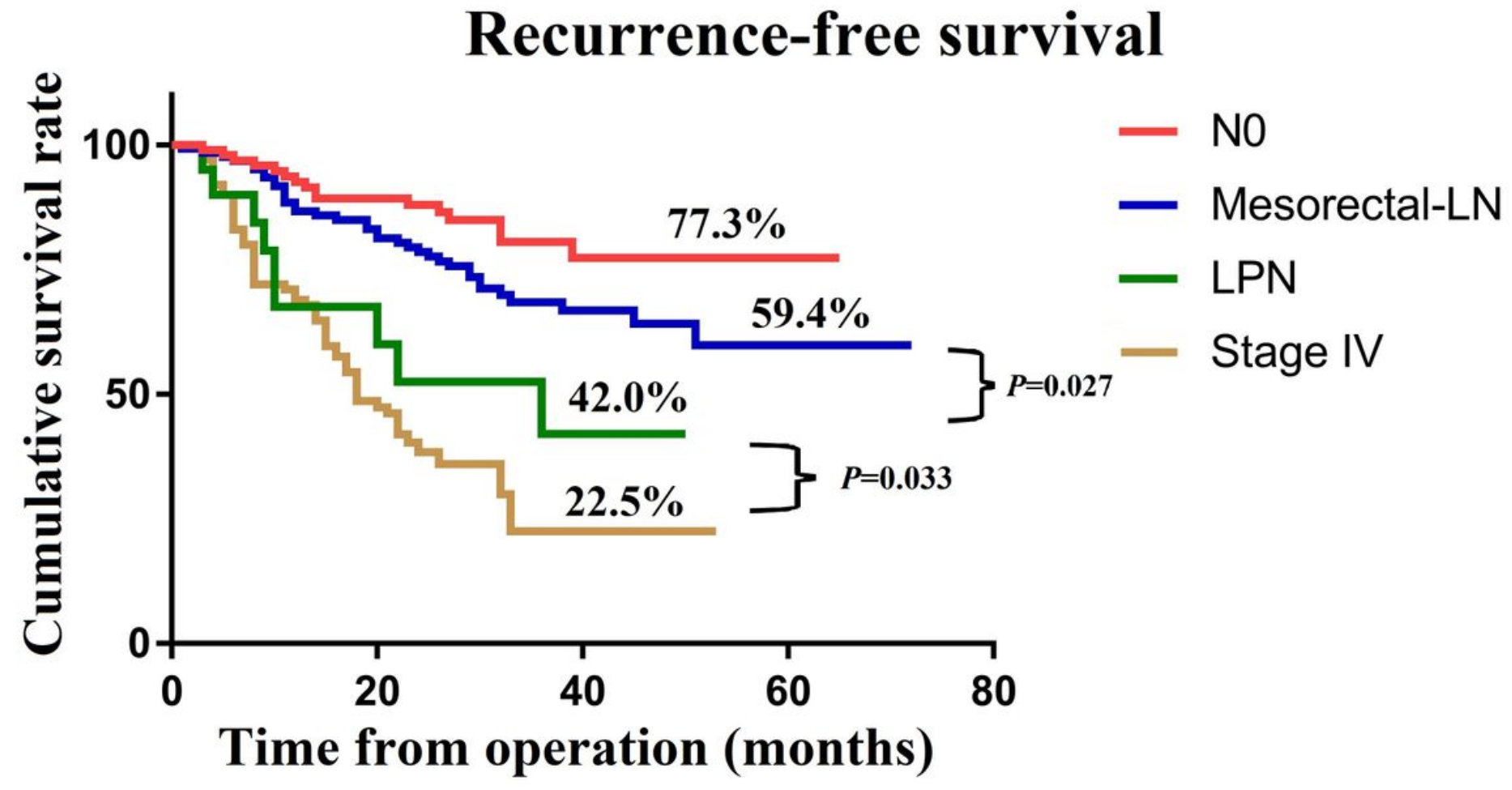

Figure 4

Recurrence-free survival for 250 patient who underwent TME with or without LPND and 100 patients with stage IV who underwent curative resection.

\section{Recurrence-free survival curve}

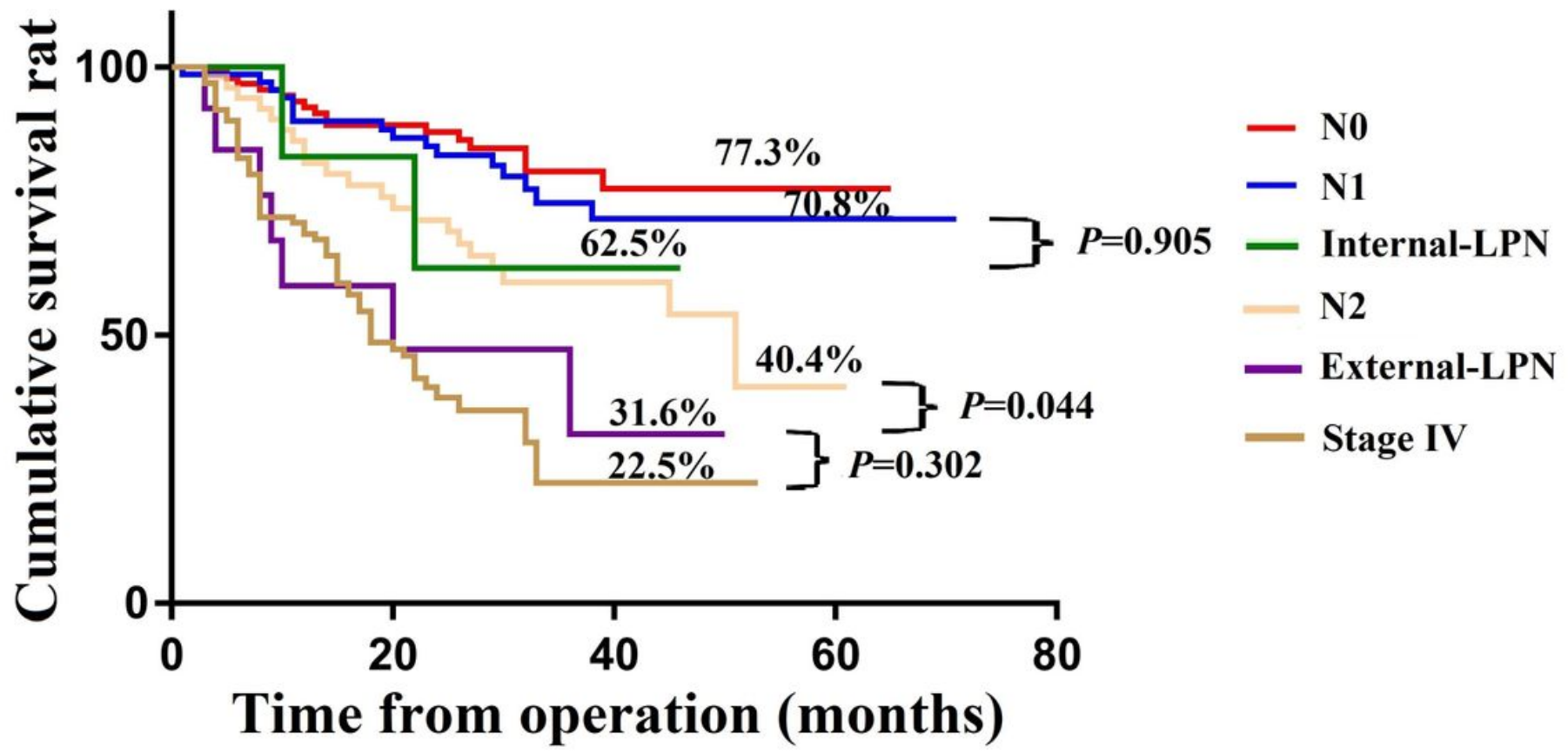




\section{Figure 5}

The recurrence-free survival for 250 patients who underwent TME with or without LPND after subgroup analysis and 100 patients with stage IV who underwent radical resection. 